\title{
Takahasi semigroups
}

\author{
Mário J. J. Branco \\ Departamento de Matemática and CAUL/CEMAT, \\ Faculdade de Ciências, Universidade de Lisboa \\ Campo Grande, 1749-016 Lisboa, Portugal \\ email: mjbranco@fc.ul.pt \\ Gracinda M. S. Gomes \\ Departamento de Matemática and CAUL/CEMAT, \\ Faculdade de Ciências, Universidade de Lisboa \\ Campo Grande, 1749-016 Lisboa, Portugal \\ email: gmcunha@fc.ul.pt \\ Pedro V. Silva \\ Centro de Matemática, Faculdade de Ciências, Universidade do Porto, \\ R. Campo Alegre 687, 4169-007 Porto, Portugal \\ email: pvsilva@fc.up.pt
}

April 2, 2015

2010 Mathematics Subject Classification: 20M99, 20M15, 37C25, 20M18

Keywords: semigroups, periodic points, periodic orbits, groups.

\begin{abstract}
Takahasi's theorem on chains of subgroups of bounded rank in a free group is generalized to several classes of semigroups. As an application, it is proved that the subsemigroups of periodic points are finitely generated and periodic orbits are bounded for arbitrary endomorphisms for various semigroups. Some of these results feature classes such as completely simple semigroups, Clifford semigroups or monoids defined by balanced one-relator presentations. In addition to the background on semigroups, proofs involve arguments over groups and finite automata.
\end{abstract}




\section{Introduction}

In a paper of 1950, Takahasi proved the following, often called Takahasi's Theorem (the same result was proved independently by Higman in the paper [10] of 1951):

Theorem 1.1. 21] Let $F$ be a free group and let $K_{1} \leqslant K_{2} \leqslant \cdots$ be an ascending chain of finitely generated subgroups of $F$. If the rank of the subgroups in the chain is bounded, then the chain is stationary.

Recall that in view of Nielsen's Theorem [15, Proposition I.2.6], every finitely generated subgroup of a free group is free, so in Takahasi's Theorem rank means the cardinality of a basis.

The concept of rank admits a natural generalization to arbitrary groups. Given a group $G$, we define its group rank, denoted by $\operatorname{rk}_{\mathcal{G}}(G)$, to be the minimum cardinality of a generating set of $G$ (as a group).

Bogopolski and Bux proved recently an analogue of Takahasi's Theorem for fundamental groups of closed compact surfaces [4, Proposition 2.2]. In [1], Araújo, Silva and Sykiotis introduced the concept of Takahasi group. A group $G$ is a Takahasi group if every ascending chain of subgroups of $G$ of bounded group rank is stationary. In [1], among other results it is proved that:

Theorem 1.2. [1, Theorem 4.1] Every finite extension of a Takahasi group is a Takahasi group.

It is also proved in [1, Section 4] that every virtually free group is a Takahasi group, and the fundamental group of a finite graph of groups with virtually polycyclic vertex groups and finite edge groups is a Takahasi group. On the other hand, in [1, Example 4.3] it is shown that a free group of finite rank has arbitrarily long strict chains of subgroups of equal rank.

Takahasi's Theorem can be applied to prove that the subgroup $\operatorname{Per}(\varphi)$ of periodic points of an automorphism $\varphi$ of a free group of finite rank is finitely generated (which implies that the size of the periodic orbits is bounded for each automorphism). Using the aforementioned generalization of Takahasi's Theorem, one obtains:

Theorem 1.3. [1, Theorem 5.1] Let $G$ be the fundamental group of a finite graph of groups with finitely generated virtually nilpotent vertex groups and finite edge groups. Then there exists a constant $M>0$ such that

$$
r k_{\mathcal{G}}(\operatorname{Per}(\varphi)) \leqslant M
$$

for every $\varphi \in \operatorname{End}(G)$.

As a consequence, a bound for the periods of a given endomorphism of $G$ was also obtained in [1].

In this paper, we consider the condition of Takahasi's Theorem in the context of several varieties of semigroups, and apply results obtained to investigate the subsemigroup of fixed points and the subsemigroup of periodic points of an endomorphism of a semigroup of various kinds.

The reader is assumed to have basic knowledge of semigroup theory, universal algebra and automata theory.

We consider the following varieties throughout this paper: 
- $\mathcal{M}$ - the variety of all monoids (type $(2,0)$ );

- $\mathcal{S}$ - the variety of all semigroups (type (2));

- $\mathcal{I}$ - the variety of all inverse semigroups (type $(2,1)$ );

- $\mathcal{G}$ - the variety of all groups (type $(2,1)$ );

- $\mathcal{C} \mathcal{R}$ - the variety of all completely regular semigroups (type $(2,1)$ );

- $\mathcal{C}$ - the variety of all Clifford semigroups (type $(2,1)$ );

- $\mathcal{C S}$ - the variety of all completely simple semigroups (type $(2,1)$ ).

The unary operation is $a \mapsto a^{-1}$ in the case of inverse semigroups and groups, where $a^{-1}$ denotes the inverse of $a$, and $a \mapsto \bar{a}$ in the case of completely regular semigroups, where $\bar{a}$ is the unique inverse of $a$ commuting with it. Recall that $\mathcal{C R}$ contains both $\mathcal{C}$ and $\mathcal{C S}$. Also

$$
\mathcal{G}=\mathcal{I} \cap \mathcal{C S}, \quad \mathcal{C}=\mathcal{I} \cap \mathcal{C R} .
$$

It is particularly important for us to remark which type of subalgebras we have for each of these varieties: submonoids for $\mathcal{M}$, subsemigroups for $\mathcal{S}$, inverse subsemigroups for $\mathcal{I}$, subgroups for $\mathcal{G}$ and completely regular subsemigroups for $\mathcal{C R}$. If $\mathcal{V}$ is any of the varieties of type $\tau$ defined above and $S \in \mathcal{V}$, we write $T \leqslant \mathcal{V} S$, and say that $T$ is a $\mathcal{V}$-subalgebra of $S$, meaning that $T$ is a $\tau$-subalgebra of $S$.

The paper is structured as follows. In Section 2, we generalize the concept of Takahasi group to further varieties of algebras. We show that finite $\mathcal{J}$-above semigroups are Takahasi, and provide a full description of Takahasi completely simple semigroups and of Takahasi Clifford semigroups. We also prove corollaries involving appropriate notions of finite index, as well as some negative results.

In Section 3, we introduce classes of semigroups UA (respectively UE) where the rank of fixed point subsemigroups is uniformly bounded for arbitrary automorphisms (respectively endomorphisms). Using the results of Section 2, we prove that the subsemigroups of periodic points are finitely generated and periodic orbits are bounded for arbitrary endomorphisms of finitely generated completely simple or Clifford semigroups whose $\mathcal{H}$-classes are Takahasi groups and belong to UE. Similar results are proved for balanced one-relator presentations of length 2 .

\section{Takahasi semigroups}

Let $S$ be a semigroup and let $A \subseteq S$ be nonempty. We denote the subsemigroup of $S$ generated by $A$ by $A^{+}$, and one has

$$
A^{+}=\bigcup_{n \geqslant 1} A^{n}
$$

If $S$ is a monoid, the submonoid of $S$ generated by $A$ will be denoted by $A^{*}$. Clearly, $A^{*}=A^{+} \cup\{1\}$. For any semigroup $S$, the rank of $S$, denoted by $\operatorname{rk}(S)$, is defined as

$$
\operatorname{rk}(S)=\min \left\{|A|: \emptyset \neq A \subseteq S, A^{+}=S\right\},
$$


if $S$ is finitely generated, and $\operatorname{ask} \operatorname{rk}(S)=\infty$ otherwise.

Assume now that $\mathcal{V}$ is an arbitrary variety. Let $S \in \mathcal{V}$ and $A \subseteq S$ be nonempty. We denote by $\langle A\rangle$ the $\mathcal{V}$-subalgebra of $S$ generated by $A$. Given $S \in \mathcal{V}$, we also define the $\mathcal{V}$-rank of $S$, denoted by $\operatorname{rk}_{\mathcal{V}}(S)$, as

$$
\operatorname{rk}_{\mathcal{V}}(S)=\min \{|A|: \emptyset \neq A \subseteq S,\langle A\rangle=S\}
$$

if $S$ is finitely generated, and $\operatorname{as~}_{\operatorname{rk}}(S)=\infty$ otherwise. Note that, for $\mathcal{V} \in\{\mathcal{M}, \mathcal{I}, \mathcal{G}, \mathcal{C R}, \mathcal{C}, \mathcal{C S}\}$, the inequalities

$$
\operatorname{rk}_{\mathcal{V}}(S) \leqslant \operatorname{rk}(S) \leqslant 2 \operatorname{rk} \mathcal{V}(S)
$$

hold for every $S \in \mathcal{V}$. Thus Takahasi's Theorem could be stated using the semigroup rank instead of the group rank.

We generalize now the concept of Takahasi group for a variety $\mathcal{V}$. Given $S \in \mathcal{V}$, we write $S \in \operatorname{Tak}(\mathcal{V})$ if every ascending chain of $\mathcal{V}$-subalgebras of $S$ of bounded $\mathcal{V}$-rank is stationary. More precisely, whenever

$$
T_{1} \leqslant T_{2} \leqslant \cdots \leqslant S
$$

and $\operatorname{rk} \mathcal{V}\left(T_{n}\right) \leqslant M$ for every $n \geqslant 1$, there exists some $p \geqslant 1$ such that $T_{p}=T_{p+1}=T_{p+2}=\cdots$

It follows easily from the definitions and (1) that

$$
\operatorname{Tak}(\mathcal{S}) \cap \mathcal{G} \subseteq \operatorname{Tak}(\mathcal{G})
$$

The following result shows that the opposite inclusion is far from true. Given groups $G$ and $H$, we denote by $G *_{\mathcal{G}} H$ and $G *_{\mathcal{S}} H$ the free product of $G$ and $H$ in the varieties $\mathcal{G}$ and $\mathcal{S}$, respectively.

Proposition 2.1. $\quad$ (i) $\mathbb{Z} \times \mathbb{Z} \notin \operatorname{Tak}(\mathcal{S})$;

(ii) if $H$ is a nontrivial group, then $\mathbb{Z} *_{\mathcal{G}} H \notin \operatorname{Tak}(\mathcal{S})$;

(iii) if $H$ is a nontrivial group, then $\mathbb{Z} *_{\mathcal{S}} H \notin \operatorname{Tak}(\mathcal{S})$.

Proof. (i) The group $\mathbb{Z} \times \mathbb{Z}$ is generated by $a=(1,0)$ and $b=(0,1)$. For every $n \geqslant 1$, let $S_{n}$ be the subsemigroup of $\mathbb{Z} \times \mathbb{Z}$ generated by $a^{-2}$ and $a^{2 n-1} b$. Since $a^{2 n-1} b=a^{-2}\left(a^{2 n+1} b\right)$ for every $n \geqslant 1$, then

$$
S_{1} \subseteq S_{2} \subseteq S_{3} \subseteq \cdots
$$

Suppose that $a^{2 n+1} b \in S_{n}$. Then the generator $a^{2 n-1} b$ must be used exactly once to get $a^{2 n+1} b$, which is clearly impossible. Hence $a^{2 n+1} b \notin S_{n}$ and so all the inclusions in (2) are strict. Therefore $\mathbb{Z} \times \mathbb{Z} \notin \operatorname{Tak}(\mathcal{S})$.

(ii) We use the same construction taking $a$ to be a generator of $\mathbb{Z}$ and $b \in H \backslash\{1\}$. Once again, we have (2).

Suppose that $a^{2 n+1} b \in S_{n}$. Then

$$
a^{2 n+1} b=a^{-2 k_{0}} a^{2 n-1} b a^{-2 k_{1}} a^{2 n-1} b \cdots a^{-2 k_{m-1}} a^{2 n-1} b a^{-2 k_{m}}
$$

for some $m \geqslant 1$ and $k_{0}, \ldots, k_{m} \geqslant 0$. Since there exists always an odd number of $a$ 's between any two consecutive $b$ 's in the right hand side, it follows easily from the normal form for the elements 
of the free product $\mathbb{Z} *_{\mathcal{G}} H$ that we must have $m=1, k_{1}=0$ and $k_{0}=-1$, a contradiction. Hence $a^{2 n+1} b \notin S_{n}$ and so all the inclusions in $(2)$ are strict. Therefore $\mathbb{Z} * \mathcal{G} H \notin \operatorname{Tak}(\mathcal{S})$.

(iii) The proof of (ii) holds for $\mathbb{Z} *_{\mathcal{S}} H$ as well.

Since $\mathbb{Z} \times \mathbb{Z}$ and the groups of the form $\mathbb{Z} *_{\mathcal{G}} H$ with $H$ finite are Takahasi groups by [1, Corollary 4.4], it follows that $\operatorname{Tak}(\mathcal{G}) \nsubseteq \operatorname{Tak}(\mathcal{S})$. By Proposition 2.1(ii), no free group of rank $>1$ belongs to $\operatorname{Tak}(\mathcal{S})$.

Another consequence of this last proposition is the bad behaviour of $\operatorname{Tak}(\mathcal{S})$ with respect to direct products and free products. But first we discuss the case of infinite cyclic groups.

Proposition 2.2. The additive semigroup $\mathbb{Z}$ belongs to $\operatorname{Tak}(\mathcal{S})$, and so does $(\mathbb{N},+)$.

For the proof we need some classical tools. Given a subsemigroup $S$ of the additive semigroup $\mathbb{N}$ of natural numbers and $d \in \mathbb{N}$, we say that $S$ is ultimately a $d$-segment if there is $p \in \mathbb{N}$ such that for all $n \in \mathbb{N}$ such that $n \geqslant p$, we have $n \in S$ if and only if $d$ divides $n$. It is clear that $S$ cannot be ultimately a $d_{1}$-segment and ultimately a $d_{2}$-segment for distinct natural numbers $d_{1}$ and $d_{2}$. Let

$$
d_{S}=\min \{\operatorname{gcd}\{x, y\}: x, y \in S\} .
$$

The next result can be found in [9, Sec. II.4] (see also [13, 20]).

Theorem 2.3. If $S$ is a subsemigroup of $\mathbb{N}$, then $S \subseteq \mathbb{N} d_{S}, S$ is ultimately a $d_{S}$-segment, and $S$ is finitely generated.

For a subsemigroup $S$ of $\mathbb{N}$, define

$$
p_{S}=\min \left\{p \in \mathbb{N}: \forall n \geqslant p\left(d_{S} \mid n \Rightarrow n \in S\right)\right\},
$$

which is a natural number by Theorem 2.3 .

From Theorem 2.3 and its dual for $\mathbb{Z}^{-}$we can easily deduce the following corollary, which can also be found in [9, Sec. II.4].

Corollary 2.4. A subsemigroup of $\mathbb{Z}$ either contains only non-negative integers, or contains only non-positive integers, or is of the form $\mathbb{Z} d$ for some $d \in \mathbb{N}$, hence a subgroup of $\mathbb{Z}$.

Now we are able to make the proof of Proposition 2.2.

Proof of Proposition 2.2. First we prove that any infinite ascending chain of subsemigroups of $\mathbb{N}$

$$
S_{1} \leqslant S_{2} \leqslant \cdots
$$

is stationary (this implies that $\mathbb{N} \in \operatorname{Tak}(\mathcal{S})$ ). Let us take such a chain. Then

$$
d_{S_{1}} \geqslant d_{S_{2}} \geqslant \cdots
$$

whence there is $k \in \mathbb{N}$ such that $d_{S_{k}}=d_{S_{i}}$ for all $i \geqslant k$. Given $i \geqslant k$, the fact that $S_{i} \leqslant S_{i+1}$ and $d_{S_{i}}=d_{S_{i+1}}$ implies that $p_{S_{i+1}} \leqslant p_{S_{i}}$. Thus

$$
p_{S_{k}} \geqslant p_{S_{k+1}} \geqslant \cdots,
$$


and then there is $\ell \geqslant k$ such that $p_{S_{\ell}}=p_{S_{i}}$ for every $i \geqslant \ell$. Then in the chain

$$
S_{\ell} \leqslant S_{\ell+1} \leqslant \cdots
$$

any two of these semigroups only may differ in natural numbers less than $p_{\ell}$, and, hence, this chain is stationary.

Dually $\mathbb{Z}^{-}$satisfies the same condition. Now let

$$
S_{1} \leqslant S_{2} \leqslant \cdots
$$

be an infinite ascending chain of nontrivial subsemigroups of $\mathbb{Z}$. By Corollary 2.4, either all these subsemigroups are contained in $\mathbb{Z}_{0}^{-}$, or all these subsemigroups are contained in $\mathbb{N}$, or there is $k \in \mathbb{N}$ such that $S_{i}$ is a subgroup of $\mathbb{Z}$ for every $i \geqslant k$. In the first two situations, the chain is stationary as we proved above. In the third situation, the claim follows immediately from $\mathbb{Z}$ being a noetherian ring. Therefore $\mathbb{Z} \in \operatorname{Tak}(\mathcal{S})$.

As opposed to Proposition 2.2, we have the following.

Proposition 2.5. The additive group $\mathbb{Q}$ of rational numbers is not a Takahasi group.

Proof. It suffices to observe that, defining, for each positive integer $k$, the subgroup $H_{k}$ as being the cyclic subgroup of $\mathbb{Q}$ generated by $\frac{1}{2^{k}}$, we have the infinite ascending chain

$$
H_{1}<H_{2}<H_{3}<\cdots
$$

of subgroups of $\mathbb{Q}$ of rank 1 .

A celebrated result of Group Theory, attributed to Higman, Neumann and Neumann, and, independently to Freudenthall, states that every countable group is embeddable in a 2-generator group [7, 11, 17]. Thus, by Proposition 2.5, there are finitely generated groups that are not Takahasi groups.

Now we can prove:

Proposition 2.6. $\operatorname{Tak}(\mathcal{S})$ is not closed under:

(i) direct product;

(ii) free product.

Proof. (i) This follows from Proposition 2.1(i) and Proposition 2.2,

(ii) Trivially, all finite semigroups belong to $\operatorname{Tak}(\mathcal{S})$. Now, the result follows from Proposition 2.1(iii) and Proposition 2.2.

On the positive side, the following result provides a wide class of examples of semigroups in $\operatorname{Tak}(\mathcal{S})$.

The quasi-order $\leqslant \mathcal{J}$ on $S$ is defined by

$$
a \leqslant \mathcal{J} b \quad \text { if } a \in S^{1} b S^{1} .
$$


A semigroup $S$ is finite $\mathcal{J}$-above if

$$
\{x \in S: x \geqslant \mathcal{J} a\}
$$

is finite for every $a \in S$.

Theorem 2.7. Let $S$ be a finite $\mathcal{J}$-above semigroup. Then $S \in \operatorname{Tak}(\mathcal{S})$.

Proof. Let $S$ be a finite $\mathcal{J}$-above semigroup and suppose that

$$
T_{1}<T_{2}<T_{3}<\cdots
$$

is an infinite ascending chain of finitely generated subsemigroups of $S$. It suffices to show that $\operatorname{rk}\left(T_{n}\right)$ is unbounded.

For each $n \geqslant 1$, we fix a generating set $A_{n}$ of $T_{n}$ of minimum size. Let $a \in A_{1}$. Since $a \in T_{n}=A_{n}^{+}$ for every $n \geqslant 1$, we have $a \in a_{n} T_{n}^{1}$ for some $a_{n} \in A_{n}$. Hence $a_{n} \geqslant \mathcal{J} a$ in $S$ for every $n \geqslant 1$. Since $S$ is finite $\mathcal{J}$-above, it follows that there exists a refinement

$$
T_{i_{1}}<T_{i_{2}}<T_{i_{3}}<\cdots
$$

of (4) and some

$$
b_{1} \in A_{i_{1}} \cap A_{i_{2}} \cap A_{i_{3}} \cap \cdots
$$

Proceeding inductively, we assume now that there exists a refinement

$$
T_{j_{1}}<T_{j_{2}}<T_{j_{3}}<\cdots
$$

of (4) and some distinct

$$
b_{1}, \ldots, b_{n} \in A_{j_{1}} \cap A_{j_{2}} \cap A_{j_{3}} \cap \cdots
$$

Since $T_{j_{1}} \subset T_{j_{2}}$, there exists some $c \in A_{j_{2}} \backslash T_{j_{1}}$. Since $c \in T_{j_{n}} \backslash T_{j_{1}}=A_{j_{n}}^{+} \backslash A_{j_{1}}^{+}$for every $n \geqslant 2$, we have $c \in T_{j_{n}}^{1} c_{n} T_{j_{n}}^{1}$ for some $c_{n} \in A_{j_{n}} \backslash A_{j_{1}}$. Hence $c_{n} \geqslant \mathcal{J} c$ in $S$ for every $n \geqslant 2$. Since $S$ is finite $\mathcal{J}$-above, it follows that there exists a refinement

$$
T_{k_{1}}<T_{k_{2}}<T_{k_{3}}<\cdots
$$

of (5) (and therefore of (4)) and some

$$
b_{n+1} \in\left(A_{k_{1}} \cap A_{k_{2}} \cap A_{k_{3}} \cap \cdots\right) \backslash A_{j_{1}} .
$$

Since $b_{1}, \ldots, b_{n} \in A_{j_{1}}$, it follows that $b_{1}, \ldots, b_{n+1}$ are $n+1$ distinct elements of $A_{k_{1}} \cap A_{k_{2}} \cap A_{k_{3}} \cap \cdots$. By induction, such a property holds for arbitrary $n$. In particular, for every $n \geqslant 1$, there exists some $m \geqslant 1$ such that $\left|A_{m}\right| \geqslant n$. Thus $\operatorname{rk}\left(T_{n}\right)=\left|A_{n}\right|$ is unbounded and so $S \in \operatorname{Tak}(\mathcal{S})$.

A semigroup (monoid) presentation of the form $\left\langle A \mid u_{i}=v_{i}(i \in I)\right\rangle$ is said to be balanced if $\left|u_{i}\right|=\left|v_{i}\right|$ for every $i \in I$.

Since the semigroups in the statement of the next corollary are clearly finite $\mathcal{J}$-above, we immediately get: 
Corollary 2.8. The following semigroups belong to $\operatorname{Tak}(\mathcal{S})$ :

(i) finite semigroups;

(ii) free semigroups and free monoids;

(iii) trace monoids;

(iv) semigroups or monoids defined by balanced presentations;

(v) free inverse semigroups and free inverse monoids.

Since $\operatorname{Tak}(\mathcal{S}) \cap \mathcal{I} \subseteq \operatorname{Tak}(\mathcal{I})$, we get also:

Corollary 2.9. Free inverse semigroups and free inverse monoids belong to Tak $(\mathcal{I})$.

We consider next $\mathcal{C S}$ and Rees matrix semigroups. But first we need a lemma on ranks of groups defined by automata. Let $A$ be an alphabet. We denote by $A^{-1}$ a set of formal inverses of $A$. If $M$ is a monoid of type $(2,1)$, and $x \mapsto x^{-1}$ is the unary operation, we say that a monoid homomorphism $\varphi:\left(A \cup A^{-1}\right)^{*} \rightarrow M$ is matched if $a^{-1} \varphi=(a \varphi)^{-1}$ holds for every $a \in A$.

We say that $\mathcal{A}=\left(Q, q_{0}, T, E\right)$ is a finite A-automaton if $Q$ is a finite set, $q_{0} \in Q, T \subseteq Q$ and $E \subseteq Q \times A \times Q$, and refer to the elements of $Q$ and $E$ as vertices and edges, respectively.

We say that an $\left(A \cup A^{-1}\right)$-automaton $\mathcal{A}=\left(Q, q_{0}, T, E\right)$ is:

- dual if

$$
(p, a, q) \in E \Leftrightarrow\left(q, a^{-1}, p\right) \in E
$$

holds for all $p, q \in Q$ and $a \in A$;

- inverse if it is dual, trim and deterministic;

- Stallings if it is inverse, $T=\left\{q_{0}\right\}$ and the unique vertex which may have outdegree 1 is $q_{0}[2$, Section 2].

Recall that the language recognized by $\mathcal{A}$ is the set $L(\mathcal{A})$ of words $w \in\left(A \cup A^{-1}\right)^{*}$ such that $w$ is the label of a path from $q_{0}$ to some $t \in T$.

Lemma 2.10. Let $A$ be a finite alphabet and let $\varphi:\left(A \cup A^{-1}\right)^{*} \rightarrow G$ be a matched homomorphism onto a group. Let $\mathcal{A}=\left(Q, q_{0}, T, E\right)$ be a finite $\left(A \cup A^{-1}\right)$-automaton such that $(L(\mathcal{A})) \varphi=G$. Then:

(i) $\operatorname{rk}_{\mathcal{G}}(G) \leqslant|E|$;

(ii) $\operatorname{rk}_{\mathcal{G}}(G) \leqslant|E|-|Q|+\left|\left\{q_{0}\right\} \cup T\right|$ if $\mathcal{A}$ is trim.

Proof. Since the trim part of $\mathcal{A}$ (i.e. the subautomaton induced by all vertices lying in some successful path) has at most $|E|$ edges, it is enough to consider the case when $\mathcal{A}$ is trim.

Let $\mathcal{A}_{1}=\left(Q_{1}, q_{0}, q_{0}, E_{1}\right)$ be the automaton obtained by identifying all the vertices of $T$ with $q_{0}$. Clearly, $\mathcal{A}_{1}$ is trim. Then let $\mathcal{A}_{2}=\left(Q_{1}, q_{0}, q_{0}, E_{2}\right)$ be the automaton obtained from $\mathcal{A}_{1}$ by adding 
edges of the form $p \stackrel{a^{-1}}{\longrightarrow} q$ whenever $E_{1}$ contains an edge $q \stackrel{a}{\longrightarrow} p\left(a \in A \cup A^{-1}\right)$ but no edge $p \stackrel{a^{-1}}{\longrightarrow} q$. Note that $\mathcal{A}_{2}$ is a trim dual automaton.

Next let $\mathcal{A}_{3}=\left(Q_{3}, q_{0}, q_{0}, E_{3}\right)$ be the inverse automaton obtained by successively identifying all pairs of edges of the form

$$
q \stackrel{a}{\longleftarrow} p \stackrel{a}{\longrightarrow} r
$$

with $a \in A \cup A^{-1}$. This is the procedure known as Stallings foldings. It is well known that the final result is independent of the order in which foldings are executed (see [2, Section 2]).

Finally, let $\mathcal{A}_{4}=\left(Q_{4}, q_{0}, q_{0}, E_{4}\right)$ be the Stallings automaton obtained by successively removing from $\mathcal{A}_{3}$ all vertices of outdegree 1 that are distinct from $q_{0}$.

We prove that

$$
\left(L\left(\mathcal{A}_{4}\right)\right) \varphi=\left(L\left(\mathcal{A}_{3}\right)\right) \varphi=\left(L\left(\mathcal{A}_{2}\right)\right) \varphi=\left(L\left(\mathcal{A}_{1}\right)\right) \varphi=(L(\mathcal{A})) \varphi=G .
$$

We start with the equality $\left(L\left(\mathcal{A}_{1}\right)\right) \varphi=\left(L\left(\mathcal{A}_{0}\right)\right) \varphi$. Clearly, $L(\mathcal{A}) \subseteq L\left(\mathcal{A}_{1}\right)$, and so $(L(\mathcal{A})) \varphi \subseteq$ $\left(L\left(\mathcal{A}_{1}\right)\right) \varphi$. To prove the opposite inclusion, it suffices to assume that we are identifying $q_{0}$ with a single element $t \in T$.

We claim that

$$
\text { if } p \stackrel{v}{\longrightarrow} q \text { is a path in } \mathcal{A} \text { and } p, q \in\left\{q_{0}, t\right\} \text {, then } v \varphi \in G \text {. }
$$

Since $\mathcal{A}$ is trim, there exists some path $q_{0} \stackrel{u}{\longrightarrow} t$ in $\mathcal{A}$. If $p=t$ and $q=q_{0}$, then $u v u \in L(\mathcal{A})$ and so $v \varphi=(u \varphi)^{-1}(u v u) \varphi(u \varphi)^{-1} \in G$. The other cases are straightforward variations of this one and can be omitted. Thus $(7)$ holds.

Now let $w \in L\left(\mathcal{A}_{1}\right)$. Then we may factor $w=w_{0} w_{1} \cdots w_{n}$ so that

$$
q_{0} \stackrel{w_{0}}{\longrightarrow} q_{0} \stackrel{w_{1}}{\longrightarrow} \cdots \stackrel{w_{n}}{\longrightarrow} q_{0}
$$

enhances all the occurrences of the vertex $q_{0}$ in a path of $\mathcal{A}_{1}$ labelled by $w$. It follows that there are paths $p_{i} \stackrel{w_{i}}{\longrightarrow} r_{i}$ in $\mathcal{A}$ with $p_{i}, r_{i} \in\left\{q_{0}, t\right\}$ for $i=0, \ldots, n$. By (7), we get $w_{i} \varphi \in G$ for every $i$, hence $w \in G=(L(\mathcal{A})) \varphi$ and so $\left(L\left(\mathcal{A}_{1}\right)\right) \varphi=(L(\mathcal{A})) \varphi$.

Similarly to the preceding equality, to prove the nontrivial inclusion $\left(L\left(\mathcal{A}_{2}\right)\right) \varphi \subseteq\left(L\left(\mathcal{A}_{1}\right)\right) \varphi$, we may assume that $\mathcal{A}_{2}$ was obtained from $\mathcal{A}_{1}$ by adding the single edge $p \stackrel{a^{-1}}{\longrightarrow} q$. Let $w \in L\left(\mathcal{A}_{2}\right) \backslash L\left(\mathcal{A}_{1}\right)$. Then we may factor $w=w_{0} a^{-1} w_{1} \cdots a^{-1} w_{n}$ so that

$$
q_{0} \stackrel{w_{0}}{\longrightarrow} p \stackrel{a^{-1}}{\longrightarrow} q \stackrel{w_{1}}{\longrightarrow} \cdots \stackrel{a^{-1}}{\longrightarrow} q \stackrel{w_{n}}{\longrightarrow} q_{0}
$$

enhances all the occurrences of the new edge in a path of $\mathcal{A}_{2}$ labelled by $w$. Since $\mathcal{A}_{1}$ is trim, there exist paths of the form

$$
q_{0} \stackrel{u}{\longrightarrow} q, \quad p \stackrel{v}{\longrightarrow} q_{0}
$$

in $\mathcal{A}_{1}$. Moreover, all the paths labelled by some $w_{i}$ in $(8)$ can be seen as paths in $\mathcal{A}_{1}$, hence $u a v, w_{0} v, u w_{n}, u w_{i} v \in L\left(\mathcal{A}_{1}\right)$ for $i=1, \ldots, n-1$ and we get

$$
w \varphi=\left(w_{0} a^{-1} w_{1} \cdots a^{-1} w_{n}\right) \varphi=\left(w_{0} v\right) \varphi((u a v) \varphi)^{-1}\left(u w_{1} v\right) \varphi \cdots((u a v) \varphi)^{-1}\left(u w_{n}\right) \varphi \in G .
$$


Thus $\left(L\left(\mathcal{A}_{2}\right)\right) \varphi=\left(L\left(\mathcal{A}_{1}\right)\right) \varphi$.

The equalities $\left(L\left(\mathcal{A}_{4}\right)\right) \varphi=\left(L\left(\mathcal{A}_{3}\right)\right) \varphi=\left(L\left(\mathcal{A}_{2}\right)\right) \varphi$ are well-known facts from the theory of Stallings foldings $([2$, Section 2]), therefore $(6)$ holds.

Let $F G_{A}$ denote the free group on $A$ and let $H$ be the subgroup of $F G_{A}$ having Stallings automaton $\mathcal{A}_{4}$ (i.e. $H$ is the canonical pre-image of $G$ in $F G_{A}$ with respect to $\varphi$ ). The famous rank formula for Stallings automata states that $\operatorname{rk}_{\mathcal{G}}(H)=\frac{\left|E_{4}\right|}{2}-\left|Q_{4}\right|+1$, see [2, Proposition 2.6]). Since $G$ is a homomorphic image of $H$, it follows that

$$
\operatorname{rk}_{\mathcal{G}}(G) \leqslant \frac{\left|E_{4}\right|}{2}-\left|Q_{4}\right|+1
$$

Now each time we delete a vertex on constructing $\mathcal{A}_{4}$ from $\mathcal{A}_{3}$, we delete at least two edges, hence

$$
\frac{\left|E_{4}\right|}{2}-\left|Q_{4}\right|+1 \leqslant \frac{\left|E_{3}\right|}{2}-\left|Q_{3}\right|+1
$$

Similarly, each time we identify two vertices on constructing $\mathcal{A}_{3}$ from $\mathcal{A}_{2}$, we identify at least two pairs of edges, hence

$$
\frac{\left|E_{3}\right|}{2}-\left|Q_{3}\right|+1 \leqslant \frac{\left|E_{2}\right|}{2}-\left|Q_{1}\right|+1 .
$$

Since $\left|E_{2}\right| \leqslant 2\left|E_{1}\right|$, condition (9) and the above inequalities yield

$$
\operatorname{rk}_{\mathcal{G}}(G) \leqslant\left|E_{1}\right|-\left|Q_{1}\right|+1
$$

Now it suffices to note that $\left|E_{1}\right| \leqslant|E|$ and $\left|Q_{1}\right|=|Q|-\left|\left\{q_{0}\right\} \cup T\right|+1$.

Let $S=M[G, I, \Lambda, P]$ be a Rees matrix semigroup. Given $X \subseteq S, i \in I$ and $\lambda \in \Lambda$, we write

$$
X^{(i \lambda)}=X \cap(\{i\} \times G \times\{\lambda\}) .
$$

Given $X \subseteq S$, we write

$$
I_{X}=\{i \in I: X \cap(\{i\} \times G \times \Lambda) \neq \emptyset\}, \quad \Lambda_{X}=\{\lambda \in \Lambda: X \cap(I \times G \times\{\lambda\}) \neq \emptyset\} .
$$

Lemma 2.11. Let $S=M[G, I, \Lambda, P]$ be a Rees matrix semigroup. Let $T \leqslant \mathcal{C S} S, i \in I_{T}$ and $\lambda \in \Lambda_{T}$. Then:

(i) $T^{(i \lambda)}$ is a subgroup of $T$ and $T^{(i \lambda)} \cong G^{(i \lambda)}$ for some $G^{(i \lambda)} \leqslant_{\mathcal{G}} G$;

(ii) if $T$ is finitely generated, then

$$
\operatorname{rk}_{\mathcal{G}}\left(T^{(i \lambda)}\right) \leqslant\left(\operatorname{rk}_{\mathcal{C S}}(T)\right)^{2}+1
$$

Proof. (i) Since $T \leqslant_{\mathcal{C R}} S$ makes $T$ a union of groups and $\{i\} \times G \times\{\lambda\}$, being an $\mathcal{H}$-class of $S$, is a group itself, then $T^{(i \lambda)}$ is a subgroup of $T$. 
Write $P=\left(p_{\lambda i}\right)$. We define a mapping

$$
\begin{aligned}
\varphi: T^{(i \lambda)} & \rightarrow G \\
(i, g, \lambda) & \mapsto g p_{\lambda i}
\end{aligned}
$$

For all $g, h \in G$, we have

$$
((i, g, \lambda)(i, h, \lambda)) \varphi=\left(i, g p_{\lambda i} h, \lambda\right) \varphi=g p_{\lambda i} h p_{\lambda i}=((i, g, \lambda) \varphi((i, h, \lambda) \varphi)
$$

hence $\varphi$ is a group homomorphism.

Since $\varphi$ is clearly injective, we get $T^{(i \lambda)} \cong T^{(i \lambda)} \varphi$, so we may take $G^{(i \lambda)}=T^{(i \lambda)} \varphi$.

(ii) Let $A$ be a generating set for $T$ (as a completely simple semigroup) of minimum size. Note that

$$
I_{T}=I_{A}, \quad \Lambda_{T}=\Lambda_{A} .
$$

We take two new elements $q_{0}, t \notin \Lambda_{A}$ and let $Q=\left\{q_{0}, t\right\} \cup \Lambda_{A}$. Let also

$$
\begin{aligned}
E & =\left\{\left(q_{0}, g, \lambda^{\prime}\right):\left(i, g, \lambda^{\prime}\right) \in A\right\} \cup\left\{\left(\lambda^{\prime}, p_{\lambda^{\prime} i^{\prime}} g, \lambda^{\prime \prime}\right): \lambda^{\prime} \in \Lambda_{A},\left(i^{\prime}, g, \lambda^{\prime \prime}\right) \in A\right\} \cup\left\{\left(\lambda, p_{\lambda i}, t\right)\right\} \\
& \subseteq Q \times G \times Q .
\end{aligned}
$$

Then $\mathcal{A}=\left(Q, q_{0}, t, E\right)$ is a finite $G$-automaton. Notions such as (successful) path and language generalize from classical automata theory to $G$-automata in the obvious way.

We prove that

$$
L(\mathcal{A})=G^{(i \lambda)}
$$

Let $g \in L(\mathcal{A})$. Then there exists a path

$$
q_{0} \stackrel{g_{0}}{\longrightarrow} \lambda_{0} \stackrel{p_{\lambda_{0} i_{1}} g_{1}}{\longrightarrow} \lambda_{1} \stackrel{p_{\lambda_{1} i_{2}} g_{2}}{\longrightarrow} \cdots \stackrel{p_{\lambda_{n-1} i_{n}} g_{n}}{\longrightarrow} \lambda_{n}=\lambda \stackrel{p_{\lambda i}}{\longrightarrow} t
$$

in $\mathcal{A}$, where $\left(i_{j}, g_{j}, \lambda_{j}\right) \in A$ for $j=0, \ldots, n, i_{0}=i$ and

$$
g=g_{0} p_{\lambda_{0} i_{1}} g_{1} p_{\lambda_{1} i_{2}} g_{2} \ldots p_{\lambda_{n-1} i_{n}} g_{n} p_{\lambda i}
$$

Hence

$$
\left(i, g p_{\lambda i}^{-1}, \lambda\right)=\left(i, g_{0}, \lambda_{0}\right)\left(i_{1}, g_{1}, \lambda_{1}\right) \cdots\left(i_{n}, g_{n}, \lambda_{n}\right) \in A^{+}=T
$$

and so $\left(i, g p_{\lambda i}^{-1}, \lambda\right) \in T^{(i \lambda)}$. Thus $g=\left(i, g p_{\lambda i}^{-1}, \lambda\right) \varphi \in T^{(i \lambda)} \varphi=G^{(i \lambda)}$ and so $L(\mathcal{A}) \subseteq G^{(i \lambda)}$.

Conversely, let $g \in G^{(i \lambda)}$. Then $\left(i, g p_{\lambda i}^{-1}, \lambda\right) \in T^{(i \lambda)}$, therefore there exist $\left(i_{0}, g_{0}, \lambda_{0}\right),\left(i_{1}, g_{1}, \lambda_{1}\right), \ldots$, $\left(i_{n}, g_{n}, \lambda_{n}\right) \in A$ such that

$$
\left(i_{0}, g_{0}, \lambda_{0}\right)\left(i_{1}, g_{1}, \lambda_{1}\right) \cdots\left(i_{n}, g_{n}, \lambda_{n}\right)=\left(i, g p_{\lambda i}^{-1}, \lambda\right)
$$

It is straightforward to check that this implies the existence of a path of the form (14) in $\mathcal{A}$. Since (15) holds as well, it follows that $g \in L(\mathcal{A})$ and so $(13)$ holds.

View $E$ as a finite alphabet and let $E^{-1}$ be a set of formal inverses of $E$. Let $\theta:\left(E \cup E^{-1}\right)^{*} \rightarrow G$ be the matched homomorphism which associates to each $e \in E$ its label. Replacing $G$ by $G^{\prime}=$ $\operatorname{Im}(\theta)$, we may assume that $\theta$ is surjective. 
Let $\mathcal{B}$ be the finite $E$-automaton obtained by replacing the label of each edge $e$ in $\mathcal{A}$ by $e$ itself. In view of $(13)$, we have $G^{(i \lambda)}=L(\mathcal{A})=(L(\mathcal{B})) \theta$. To prove that $\mathcal{A}$ is trim, we take $\lambda^{\prime} \in \Lambda_{A}$. Then $A$ contains elements of the form $\left(i, g_{1}, \lambda_{1}\right),\left(i_{2}, g_{2}, \lambda^{\prime}\right)$ and $\left(i_{3}, g_{3}, \lambda\right)$. It is easy to check that there exists a path

$$
q_{0} \stackrel{g_{1}}{\longrightarrow} \lambda_{1} \stackrel{p_{\lambda_{1} i_{2}} g_{2}}{\longrightarrow} \lambda^{\prime} \stackrel{p_{\lambda^{\prime} i_{3}} g_{3}}{\longrightarrow} \lambda \stackrel{p_{\lambda i}}{\longrightarrow} t
$$

in $\mathcal{A}$, hence $\mathcal{A}$ is trim, and so is $\mathcal{B}$.

Thus we may apply Lemma 2.10 to get

$$
\operatorname{rk}_{\mathcal{G}}\left(G^{i \lambda}\right) \leqslant|E|-|Q|+2 \leqslant|A|+\left|\Lambda_{A}\right| \cdot|A|+1-\left|\Lambda_{A}\right|=\left(\left|\Lambda_{A}\right|+1\right)(|A|-1)+2 .
$$

Since $\left|\Lambda_{A}\right| \leqslant|A|=\operatorname{rk}_{\mathcal{C S}}(T)$, we get in view of (i)

$$
\operatorname{rk}_{\mathcal{G}}\left(T^{i \lambda}\right) \leqslant\left(\operatorname{rk}_{\mathcal{C S}}(T)\right)^{2}+1
$$

as required.

Theorem 2.12. Let $S=M[G, I, \Lambda, P]$ be a Rees matrix semigroup. Then, $S \in \operatorname{Tak}(\mathcal{C S})$ if and only if $G \in \operatorname{Tak}(\mathcal{G})$.

Proof. If $H$ is a subgroup of $G$, then $H \leqslant \mathcal{C S} S$ up to isomorphism and $\operatorname{rk}_{\mathcal{C S}}(H)=\operatorname{rk}_{\mathcal{G}}(H)$. It follows that if $S \in \operatorname{Tak}(\mathcal{C S})$, then $G \in \operatorname{Tak}(\mathcal{G})$.

Conversely, assume that $G$ is a Takahasi group. Let $N \geqslant 0$ and suppose that

$$
T_{1} \leqslant T_{2} \leqslant T_{3} \leqslant \cdots
$$

is an infinite chain of completely simple subsemigroups of $S$ with $\operatorname{rk}_{\mathcal{C} S}\left(T_{n}\right) \leqslant N$ for every $n \geqslant 1$. If $\operatorname{rk}_{\mathcal{C S}}\left(T_{n}\right)$ is realized by $A_{n}$, it follows from 12 that

$$
\left|I_{T_{n}}\right|=\left|I_{A_{n}}\right| \leqslant\left|A_{n}\right|=\operatorname{rk}_{\mathcal{C S}}\left(T_{n}\right) \leqslant N .
$$

Since $I_{T_{1}} \subseteq I_{T_{2}} \subseteq \cdots$, then this chain must be stationary. Similarly, the chain $\Lambda_{T_{1}} \subseteq \Lambda_{T_{2}} \subseteq \cdots$ is stationary. Removing finitely many terms of $(16)$ if needed, we may assume that $I_{T_{n}}=I^{\prime}$ and $\Lambda_{T_{n}}=\Lambda^{\prime}$ for all $n \geqslant 1$, for some $I^{\prime}$ and $\Lambda^{\prime}$ finite.

In view of Lemma 2.11(i), for all $i \in I^{\prime}$ and $\lambda \in \Lambda^{\prime}$, we have chains of subgroups

$$
T_{1}^{(i \lambda)} \leqslant T_{2}^{(i \lambda)} \leqslant \cdots
$$

By the proof of Lemma 2.11(i), we get a chain

$$
G_{1}^{(i \lambda)} \leqslant G_{2}^{(i \lambda)} \leqslant \cdots
$$

of subgroups of $G$. Since Lemma 2.11(ii) yields

$$
\operatorname{rk}_{\mathcal{G}}\left(G_{n}^{(i \lambda)}\right)=\operatorname{rk}_{\mathcal{G}}\left(T_{n}^{(i \lambda)}\right) \leqslant N^{2}+1
$$

and $G \in \operatorname{Tak}(\mathcal{G})$, each of the chains $(18)$ (and so each of the chains $(17)$ ) must be stationary. Since $I^{\prime}$ and $\Lambda^{\prime}$ are both finite and

$$
T_{n}=\bigcup_{i \in I^{\prime}} \bigcup_{\lambda \in \Lambda^{\prime}} T_{n}^{(i \lambda)}
$$

for every $n \geqslant 1$, it follows that the chain $(16)$ is also stationary. Therefore $S \in \operatorname{Tak}(\mathcal{C S})$. 
Corollary 2.13. Let $S$ be a completely simple semigroup and let $T \leqslant \mathcal{C S} S$. Let $a \in T$ and let $G$ and $H$ be the $\mathcal{H}$-classes of a in $S$ and in $T$, respectively. If $T \in \operatorname{Tak}(\mathcal{C S})$ and $H$ is a subgroup of $G$ of finite index, then $S \in \operatorname{Tak}(\mathcal{C S})$.

Proof. By Theorem 1.2, $H \in \operatorname{Tak}(\mathcal{G})$ implies $G \in \operatorname{Tak}(\mathcal{G})$. Now the claim follows from Theorem 2.12 .

We consider next Clifford semigroups. This class of semigroups admits various different characterizations. One of them states that a semigroup $S$ is a Clifford semigroup if and only if $\mathcal{H}$ is a semilattice congruence on $S$.

Theorem 2.14. Let $S$ be a Clifford semigroup. Then, $S \in \operatorname{Tak}(\mathcal{C})$ if and only if every $\mathcal{H}$-class of $S$ is a Takahasi group.

Proof. It is clear that if $S \in \operatorname{Tak}(\mathcal{C})$, then every $\mathcal{H}$-class of $S$ is a Takahasi group.

Conversely, assume that every $\mathcal{H}$-class of $S$ is a Takahasi group. Let $H$ be an $\mathcal{H}$-class of $S$. First, we show that given $T \leqslant_{\mathcal{C}} S$ such that $T \cap H \neq \emptyset$,

$$
\operatorname{rk}_{\mathcal{G}}(T \cap H) \leqslant \operatorname{rk}_{\mathcal{C}}(T) \text {. }
$$

Let $e$ denote the identity element of $H$. Since $T \cap H \neq \emptyset$ and $T$ is a Clifford subsemigroup of $S$, then $T \cap H$ is a group of $H$. Let $T^{\prime}=\{t \in T: t e \in H\}$. We claim that

$$
t \in T^{\prime} \Leftrightarrow t e \nless \mathcal{J} e,
$$

where $<_{\mathcal{J}}$ denotes the $\mathcal{J}$-order in $T$.

Indeed, we have always $t e \leqslant \mathcal{J} e$. Since $\mathcal{J}=\mathcal{H}$ in a Clifford semigroup, we get

$$
\text { te } \nless \mathcal{J} e \Leftrightarrow t e \mathcal{J} e \Leftrightarrow t e \mathcal{H} e \Leftrightarrow t e \in H \Leftrightarrow t \in T^{\prime}
$$

and so $(20)$ holds.

If $t, u \in T^{\prime}$, then

$$
t u e=t(e u e)=(t e)(u e) \in H,
$$

hence $T^{\prime}$ is a subsemigroup of $T$.

Assume first that $T^{\prime}=T$. Then

$$
\begin{aligned}
\psi: T & \rightarrow T \cap H \\
t & \mapsto t e
\end{aligned}
$$

is a semigroup homomorphism by (21). Given $t \in T$, and since idempotents are central in a Clifford semigroup, we have

$$
(t \psi)^{-1}=(t e)^{-1}=e^{-1} t^{-1}=e t^{-1}=t^{-1} e=t^{-1} \psi,
$$

hence $\psi$ is a homomorphism of Clifford semigroups. Since $\psi$ fixes each element of $T \cap H$, then it is surjective and so

$$
\operatorname{rk}_{\mathcal{G}}(T \cap H)=\operatorname{rk}_{\mathcal{C}}(T \cap H) \leqslant \operatorname{rk}_{\mathcal{C}}(T) .
$$


Thus we may assume that $T \backslash T^{\prime} \neq \emptyset$. Let $(T \cap H)^{0}$ be the Clifford semigroup obtained by adjoining a zero element 0 to $T \cap H$. We define a mapping $\psi: T \rightarrow(T \cap H)^{0}$ by

$$
t \psi= \begin{cases}t e & \text { if } t \in T^{\prime} \\ 0 & \text { otherwise }\end{cases}
$$

Let $t, u \in T$. If $t, u \in T^{\prime}$, then $(t u) \psi=t u e=(t \psi)(u \psi)$ by (21). Suppose next that $u \notin T^{\prime}$. By (20), we have $u e<\mathcal{J} e$, hence tue $<_{\mathcal{J}}$ e. It follows that $t u \notin T^{\prime}$, whence $(t u) \psi=0=(t \psi)(u \psi)$. Finally, assume that $t \notin T^{\prime}$. Then $t e<_{\mathcal{J}}$ e by $(20)$, hence $t u e=t e u<_{\mathcal{J}} e$ and so $(t u) \psi=0=(t \psi)(u \psi)$. Thus $\psi$ is a semigroup homomorphism. Similarly to the case $T^{\prime}=T$, we show that $(t \psi)^{-1}=t^{-1} \psi$, hence $\psi$ is a homomorphism of Clifford semigroups. Since $T^{\prime} \neq T$ and $\psi$ fixes each element of $T \cap H$, then it is surjective and so

$$
\operatorname{rk}_{\mathcal{C}}\left((T \cap H)^{0}\right) \leqslant \operatorname{rk}_{\mathcal{C}}(T) .
$$

If $A$ is a generating set of minimum size for $(T \cap H)^{0}$ in $\mathcal{C}$, then $A \backslash\{0\}$ generates $T \cap H$. Therefore (19) holds.

Now let $N \geqslant 0$ and suppose that

$$
T_{1} \leqslant T_{2} \leqslant T_{3} \leqslant \cdots
$$

is an infinite chain of Clifford subsemigroups of $S$ with $\operatorname{rk}_{\mathcal{C}}\left(T_{n}\right) \leqslant N$ for every $n \geqslant 1$. Consider the canonical homomorphism $\varphi: S \rightarrow S / \mathcal{H}$ and write $Y_{n}=T_{n} \varphi$. Since the free semilattice on a set with $m$ elements has $2^{m}-1$ elements, it follows from $\operatorname{rk}_{\mathcal{C}}\left(T_{n}\right) \leqslant N$ that

$$
\left|Y_{n}\right| \leqslant 2^{N}-1
$$

Therefore the chain $Y^{\prime}=Y_{1} \subseteq Y_{2} \subseteq \cdots$ must be stationary. Removing finitely many terms of (22) if needed, we may assume that $Y_{n}=Y^{\prime}$ for every $n \geqslant 1$. Thus $Y^{\prime}$ consists of finitely many $\mathcal{H}$-classes $H_{1}, \ldots, H_{m}$ of $S$ with $m \leqslant 2^{N}-1$.

For each $i=1, \ldots, m$, we get a chain of subgroups of $H_{i}$ of the form

$$
T_{1} \cap H_{i} \leqslant T_{2} \cap H_{i} \leqslant \cdots
$$

and from 190 we have $\operatorname{rk}_{\mathcal{G}}\left(T_{n} \cap H_{i}\right) \leqslant \operatorname{rk}_{\mathcal{C}}\left(T_{n}\right) \leqslant N$ for every $n \geqslant 1$. Since $H_{i} \in \operatorname{Tak}(\mathcal{G})$, the chain (23) must be stationary for each $i$. As $Y^{\prime}$ is finite and

$$
T_{n}=\bigcup_{i=1}^{m}\left(T_{n} \cap H_{i}\right)
$$

for every $n \geqslant 1$, it follows that the chain 22 is also stationary. Therefore $S \in \operatorname{Tak}(\mathcal{C})$.

We have not succeeded so far on establishing whether a completely regular semigroup where the $\mathcal{H}$-classes are Takahasi groups belongs to $\operatorname{Tak}(\mathcal{C R})$. A first obstacle is that a finitely generated completely regular semigroup may have infinitely many $\mathcal{H}$-classes. The first such example was found by Clifford in [6, Section 6]: the free completely regular semigroup on two generators. 
We introduce now a notion of index for Clifford semigroups. Let $S$ be a Clifford semigroup with $\mathcal{H}$-classes $\left\{H_{i}: i \in I\right\}$ and let $T$ be a $(2,1)$-subalgebra of $S$. Then $T$ is also a Clifford semigroup and $T=\cup_{i \in I}\left(H_{i} \cap T\right)$. Thus, each $H_{i} \cap T$ is the empty set or a subgroup of $H_{i}$. Define the index of $T$ in $S$, which we denote by $[S: T]$, by

$$
[S: T]=\sup \left\{\left[H_{i}: H_{i} \cap T\right]: i \in I\right\},
$$

with the convention that, for any group $G,[G: \emptyset]$ is the order of $G$. Clearly, this definition does not give rise to any contradiction if $S$ and $T$ are groups. Theorem 1.2 can be generalized as follows.

Theorem 2.15. If $S$ is a Clifford semigroup with a $(2,1)$-subalgebra $T$ of finite index such that $T \in \operatorname{Tak}(\mathcal{C})$, then $S \in \operatorname{Tak}(\mathcal{C})$.

Proof. Let $S$ and $T$ as in the statement. Write $S / \mathcal{H}=\left\{H_{i}: i \in I\right\}$. Let $i \in I$. If $H_{i} \cap T=\emptyset$, then $H_{i}$ is a finite group, by hypothesis, hence a Takahasi group. Besides, since $T \in \operatorname{Tak}(\mathcal{C})$, every nonempty group $H_{i} \cap T$ belongs to $\operatorname{Tak}(\mathcal{C})$, and therefore is a Takahasi group. Thus, by Theorem 1.2, $H_{i}$ is a Takahasi group. Now Theorem 2.14 gives the desired conclusion.

Now we will compare this notion of index with a notion of index introduced by Gray and Ruskuc [8]. Let $S$ be a semigroup and let $T$ be a subsemigroup of $S$. Define the binary relation $\mathrm{E}^{T}$ on $S$ by

$$
a \mathrm{七}^{T} b \Leftrightarrow T^{1} a=T^{1} b
$$

for all $a, b \in S$. Define $\mathcal{R}^{T}$ dually, and $\mathcal{H}^{T}=\mathrm{E}^{T} \cap R^{T}$. Each of these relations is an equivalence relation on $S$ and both $T$ and $S \backslash T$ are union of $\mathrm{E}^{T}$-classes (resp. $\mathcal{R}^{T}$-classes, $\mathcal{H}^{T}$-classes). In this context, those authors have defined the Green index of $T$ in $S$, which we denote by $[S: T]_{\mathrm{Gr}}$, as $n+1$, where $n$ is the cardinal of the set of $\mathcal{H}^{T}$-classes contained in $S \backslash T$. This notion when restricted to groups $S$ and $T$ coincides with the usual notion of index of a subgroup in a group. Let us see how it relates with our notion of index in the case of Clifford semigroups.

Proposition 2.16. Let $S$ be a Clifford semigroup such that $S / \mathcal{H}$ is finite and let $T$ be a $(2,1)$ subalgebra of $S$.

$$
\text { If }[S: T]<\infty \text {, then }[S: T]_{\mathrm{Gr}}<\infty \text {. }
$$

Proof. Write $S / \mathcal{H}=\left\{H_{i}: i \in I\right\}$. Since $\mathcal{H}^{T} \subseteq \mathcal{H}$, each $\mathcal{H}$-class $H_{i}$ is a union of $\mathcal{H}^{T}$-classes.

Suppose that $H_{i} \cap T \neq \emptyset$ and let $a, b \in H_{i}$. If $\left(H_{i} \cap T\right) a=\left(H_{i} \cap T\right) b$, then $a \in T b$ and $b \in T a$, whence $a \mathrm{七}^{T} b$. Dually, if $a\left(H_{i} \cap T\right)=b\left(H_{i} \cap T\right)$, then $a \mathcal{R}^{T} b$. Let

$$
I_{1}=\left\{i \in I: H_{i} \cap T=\emptyset\right\} \quad \text { and } \quad I_{2}=I \backslash I_{1} .
$$

Let

$$
\mathcal{X}=\bigcup_{i \in I_{2}}\left\{\left(\left(H_{i} \cap T\right) a, a\left(H_{i} \cap T\right)\right): a \in H_{i}\right\} .
$$

Then it is well defined the mapping $\psi: \mathcal{X} \rightarrow S / \mathcal{H}^{T}$ such that

$$
\left(\left(H_{i} \cap T\right) a, a\left(H_{i} \cap T\right)\right) \psi=H_{a}^{T},
$$


for $i \in I_{2}$ and $a \in H_{i}$, where $H_{a}^{T}$ denotes the $\mathcal{H}^{T}$-class of $a$.

Assume that $[S: T]<\infty$. Then $H_{i}$ is finite for any $i \in I_{1}$. Moreover, $\mathcal{X}$ is finite, since $I$ is finite, and so is $[S: T]$, whence $\mathcal{X} \psi$ is finite too. Clearly

$$
S / \mathcal{H}^{T}=\left(\bigcup_{i \in I_{1}}\left\{H_{a}^{T}: a \in H_{i}\right\}\right) \cup \mathcal{X} \psi,
$$

hence $[S: T]_{\mathrm{Gr}} \leqslant\left|S / \mathcal{H}^{T}\right|<\infty$.

Next, we give an example that shows the analogue of Theorem 2.15 for Green index as well as the converse of Proposition 2.16 do not hold.

Example 2.17. Let $G_{0}$ be a finitely generated group that is not a Takahasi group (we have observed that such a group exists). Let $A$ be a finite generating set of $G_{0}$ and let $G_{1}$ be the free group over $A$. Then there is a surjective homomorphism $\phi: G_{1} \rightarrow G_{0}$. Let $S=G_{0} \bullet G_{1}$ endowed with the product that extends the products in $G_{0}$ and in $G_{1}$ and such that, for $a \in G_{0}$ and $b \in G_{1}, a \cdot b=a(b \phi)$ and $b \cdot a=(b \phi) a$. Then $S$ is a Clifford semigroup (it is a strong semilattice of groups) such that $\left[S: G_{1}\right]_{G r}=2$ and $\left[S: G_{1}\right]=\left|G_{0}\right|=\infty$. Moreover, by Theorem 1.1, $G_{1}$ is a Takahasi group. However, $S \notin \operatorname{Tak}(\mathcal{C})$ since $G_{0} \notin \operatorname{Tak}(\mathcal{C})$.

\section{$3 \quad$ Periodic points}

In this section we apply the results of Section 2 to the study of the subsemigroups of periodic points as well as of the periodic orbits of the endomorphisms of some classes of semigroups.

For technical reasons, in this section we consider the empty set to be a semigroup (of rank 0).

Let $\mathcal{V}$ be one of the varieties considered in Section 1. Given $S \in \mathcal{V}$ we denote by $\operatorname{Aut}(S)$ (respectively $\operatorname{End}(S)$ ) the automorphism group (respectively endomorphism monoid) of $S$. Note that, when dealing with homomorphisms, for the varieties of type $(2,1)$ there is no need to specify the unary operation: any semigroup homomorphism between inverse (respectively completely regular) semigroups preserves necessarily the respective unary operation.

Given $\varphi \in \operatorname{End}(S)$, its fixed point subsemigroup is

$$
\operatorname{Fix}(\varphi)=\{a \in S: a \varphi=a\}
$$

and its periodic point subsemigroup is

$$
\operatorname{Per}(\varphi)=\bigcup_{n \geqslant 1} \operatorname{Fix}\left(\varphi^{n}\right)
$$

Notice that $\operatorname{Fix}(\varphi)$ and $\operatorname{Per}(\varphi)$ are actually $\mathcal{V}$-subalgebras of $S$.

Given $x \in \operatorname{Per}(\varphi)$, the period of $x$ is the least $n \geqslant 1$ such that $x \varphi^{n}=x$.

Let $\mathbf{U A}(\mathcal{V})$ denote the class of all $S \in \mathcal{V}$ such that

$$
\exists N \in \mathbb{N}, \forall \varphi \in \operatorname{Aut}(S), \quad \operatorname{rk}(\operatorname{Fix}(\varphi)) \leqslant N .
$$


Similarly, we denote by $\mathbf{U E}(\mathcal{V})$ the class of all $S \in \mathcal{V}$ such that

$$
\exists N \in \mathbb{N}, \forall \varphi \in \operatorname{End}(S), \quad \operatorname{rk}(\operatorname{Fix}(\varphi)) \leqslant N .
$$

Clearly, $\mathbf{U E}(\mathcal{V}) \subseteq \mathbf{U A}(\mathcal{V})$. By considering the identity automorphism, every $S \in \mathbf{U A}(\mathcal{V})$ must be finitely generated. Note that, in view of (1), the definitions of $\mathbf{U A}(\mathcal{V})$ and $\mathbf{U E}(\mathcal{V})$ would not be affected if we had replaced $\mathrm{rk}$ by $\mathrm{rk}_{\mathcal{V}}$. In the case that $\mathcal{V}$ is one of the varieties of type $(2,1)$, if $S \in \mathcal{V}$, then $\operatorname{Aut}(S)$ (respectively $\operatorname{End}(S)$ ) is formed by all semigroup automorphisms (respectively semigroup endomorphisms) of $S$, as we had observed. Thus, in this case we will refer to the classes $\mathbf{U A}(\mathcal{V})$ and $\mathbf{U E}(\mathcal{V})$ simply as $\mathbf{U A}$ and $\mathbf{U E}$, respectively. Observe, however, that semigroup homomorphisms between monoids do not necessarily respect the identity.

Let $F G_{n}$ denote the free group of rank $n \in \mathbb{N}$. Using their sophisticated train track techniques, Bestvina and Handel proved in [3] that, for every $\varphi \in \operatorname{Aut}\left(F G_{n}\right)$,

$$
\operatorname{rk}(\operatorname{Fix}(\varphi)) \leqslant n
$$

Latter, Imrich and Turner used this fact to prove in [12] that the same relation holds for every $\varphi \in \operatorname{End}\left(F G_{n}\right)$. Thus $F G_{n} \in \mathbf{U E}$ for every $n \in \mathbb{N}$.

More generally, as stated in Theorem 1.3, fundamental groups of finite graphs of groups with finitely generated virtually nilpotent vertex groups and finite edge groups belong to UE.

For semigroups, we should mention that in the proof of [18, Theorem 3.1] it was shown that, whenever $\varphi$ is an endomorphism of a finitely generated trace monoid (i.e. partially commutative monoid) $\mathbb{M}(A, I)$, we have $\operatorname{rk}_{\mathcal{M}}(\operatorname{Fix}(\varphi)) \leqslant 2^{|A|}$. Therefore $\mathbb{M}(A, I) \in \mathbf{U E}(\mathcal{M})$ if $A$ is finite.

Next, in a series of results, we provide some more instances of semigroups in UE.

Lemma 3.1. Let $S$ be a completely regular semigroup with finitely many $\mathcal{H}$-classes. If all $\mathcal{H}$-classes of $S$ are in $\mathbf{U E}$, then $S \in \mathbf{U E}$.

Proof. Let $H_{1}, \ldots, H_{n}$ be the $\mathcal{H}$-classes of $S$. For $i=1, \ldots, n$, assume that

$$
\operatorname{rk}(\operatorname{Fix}(\psi)) \leqslant N_{i} \quad \text { for every } \psi \in \operatorname{End}\left(H_{i}\right) .
$$

We show that

$$
\operatorname{rk}(\operatorname{Fix}(\varphi)) \leqslant \sum_{i=1}^{n} N_{i}
$$

for every $\varphi \in \operatorname{End}(S)$.

Fix $\varphi \in \operatorname{End}(S)$ and let

$$
I=\left\{i \in\{1, \ldots, n\}: \operatorname{Fix}(\varphi) \cap H_{i} \neq \emptyset\right\} .
$$

If $i \in I$, then $H_{i} \varphi \cap H_{i} \neq \emptyset$, and this yields $H_{i} \varphi \subseteq H_{i}$ since the $\mathcal{H}$-classes are the maximal subgroups of $S \in \mathcal{C} \mathcal{R}$. For every $i \in I$, let $\varphi_{i}$ denote the restriction of $\varphi$ to $H_{i}$, which is itself an endomorphism. It is immediate that

$$
\operatorname{Fix}(\varphi)=\bigcup_{i \in I} \operatorname{Fix}\left(\varphi_{i}\right)
$$


In view of (24), we get

$$
\operatorname{rk}(\operatorname{Fix}(\varphi)) \leqslant \sum_{i \in I} \operatorname{rk}\left(\operatorname{Fix}\left(\varphi_{i}\right)\right) \leqslant \sum_{i \in I} N_{i} \leqslant \sum_{i=1}^{n} N_{i}
$$

and so 25 holds. Therefore $S \in \mathbf{U E}$.

Proposition 3.2. The following semigroups belong to UE:

(i) finitely generated completely simple semigroups with $\mathcal{H}$-classes in $\mathbf{U E}$;

(ii) finitely generated Clifford semigroups with $\mathcal{H}$-classes in $\mathbf{U E}$.

Proof. (i) Let $S=M[G, I, \Lambda, P]$ be finitely generated. Then both $I$ and $\Lambda$ must be finite. Thus $S$ has finitely many $\mathcal{H}$-classes and the claim follows from Lemma 3.1 .

(ii) Let $S \in \mathcal{C}$. Then the canonical mapping $S \rightarrow S / \mathcal{H}$ is a surjective homomorphism, and $S / \mathcal{H}$ is a semilattice. Thus, if $S$ is finitely generated, then $S / \mathcal{H}$ is also finitely generated. Since finitely generated semilattices are well known to be finite, we may now apply Lemma 3.1 .

It was noticed in [1] that there exist automorphisms $\varphi$ of the group $F G_{2} \times \mathbb{Z}$ such that neither $\operatorname{Fix}(\varphi)$ nor $\operatorname{Per}(\varphi)$ is finitely generated as a group. Hence $F G_{2} \times \mathbb{Z} \notin \mathbf{U A}$. Now we give an example of a finite $\mathcal{J}$-above semigroup which satisfies the analogous property.

Example 3.3. Let $S$ be the semigroup defined by the presentation $\langle a, b, c \mid c a c=c b c\rangle$. This semigroup is finite $\mathcal{J}$-above, since $|c a c|=|c b c|$. Clearly, there exists an endomorphism $\varphi$ of $S$ satisfying $a \varphi=b, b \varphi=a$ and $c \varphi=c$. Since $\varphi^{2}=\mathrm{id}_{S}$, this homomorphism is an automorphism of $S$. The elements of $S$

$$
\text { cac, }(c a)^{2} c,(c a)^{3} c, \ldots
$$

are pairwise distinct and belong to $\operatorname{Fix}(\varphi)$. By definition of $S$, given $n \in \mathbb{N}$, the nontrivial factorizations of $(c a)^{n} c$ in $S$ of length two are $(c a)^{n} c=u v$, where

$$
(u, v)=\left((c a)^{k} c,(a c)^{n-k}\right) \quad \text { or } \quad(u, v)=\left((c a)^{k+1},(c a)^{n-k-1} c\right),
$$

with $k \in\{0, \ldots, n-1\}$. However, in any of these situations, $\{u, v\} \nsubseteq \operatorname{Fix}(\varphi)$. Then any generating set of $\operatorname{Fix}(\varphi)$ contains cac, $(c a)^{2} c,(c a)^{3} c, \ldots$, and hence $\operatorname{Fix}(\varphi)$ is not finitely generated. Therefore $S \notin \mathbf{U A}$. Notice that $\operatorname{Per}(\varphi)=\operatorname{Fix}(\varphi)$, since $\varphi^{2}=\varphi$.

The following result and its corollary show that the above counterexample is in some sense minimal among the semigroups not in UA defined by one-relator balanced presentations.

Theorem 3.4. Let $M$ be the monoid defined by a finite presentation of the form

$$
\left\langle A \mid a_{1} a_{2}=a_{3} a_{4}\right\rangle
$$

with $a_{1}, \ldots, a_{4} \in A$ not necessarily distinct. Let $\varphi \in \operatorname{End}(M)$. Then

$$
\operatorname{rk}_{\mathcal{M}}(\operatorname{Fix}(\varphi)) \leqslant|A| .
$$


Proof. We use induction on $|A|$. The case $|A|=1$ is trivial, since $\operatorname{Fix}(\varphi)=\{1\}$ or $\operatorname{Fix}(\varphi)=M$. Now assume that $|A|>1$ and the claim holds for smaller alphabets.

The possibility of induction is legitimate since any submonoid of $M$ generated by a proper subset of $A$ can still be defined by a presentation of the form (26) as we prove next. Let $A^{\prime}$ be such a subset, and let $M^{\prime}$ be the submonoid of $M$ generated by $A^{\prime}$. If $a_{1}, \ldots, a_{4} \in A^{\prime}$, then it is easy to see that $M^{\prime}$ is presented by $\left\langle A^{\prime} \mid a_{1} a_{2}=a_{3} a_{4}\right\rangle$, and $M$ is the free product of $M^{\prime}$ and the free monoid on $A \backslash A^{\prime}$. Assume now that $\left\{a_{1}, \ldots, a_{4}\right\} \not A^{\prime}$.

Suppose that $a_{1} \neq a_{3}$ and $a_{2} \neq a_{4}$. If we view (26) as a group presentation, it becomes a onerelator presentation with a cyclically reduced relator $a_{1} a_{2} a_{4}^{-1} a_{3}^{-1}$. By Magnus' famous Freiheitssatz (see [16]), since the subgroup generated by $A^{\prime}$ misses one of the generators occurring in the cyclically reduced relator, it is the free group on $A^{\prime}$. Now it follows easily that $M^{\prime}$ is the free monoid on $A^{\prime}$, hence trivially definable by a presentation of the form $(26)$.

Finally, by left-right symmetry, we only need to consider the case of presentations of the form $\left\langle A \mid a b=a^{2}\right\rangle$ or $\langle A \mid a b=a c\rangle$. In the first case, we may still use the Freiheitssatz since $b a^{-1}$ is a cyclically reduced relator where $a$ and $b$ both occur. The second case follows easily from the fact that there are no nontrivial overlappings involving $a b$ and $a c$, thus every application of the relation $a b=a c$ (involving a letter which is not in $A^{\prime}$ ) must be "undone" the exact same way. That is, $M^{\prime}$ is the free monoid on $A^{\prime}$.

Let $A_{f}$ be the set of letters of $A$ occurring in any word representing any fixed point of $\varphi$. Let $M^{\prime}$ denote the submonoid of $M$ generated by $A_{f}$. Then $u=u \varphi$ for all $u \in \operatorname{Fix}(\varphi)$ yields $A_{f} \varphi \subseteq M^{\prime}$, and so the restriction $\varphi^{\prime}=\left.\varphi\right|_{M^{\prime}}$ is an endomorphism of $M^{\prime}$. Moreover, $\operatorname{Fix}(\varphi)=\operatorname{Fix}\left(\varphi^{\prime}\right)$.

If $A_{f} \subset A$, we get

$$
\operatorname{rk}_{\mathcal{M}}(\operatorname{Fix}(\varphi))=\operatorname{rk}_{\mathcal{M}}\left(\operatorname{Fix}\left(\varphi^{\prime}\right)\right) \leqslant\left|A_{f}\right|<|A|
$$

by the induction hypothesis. Now assume that $A_{f}=A$.

Before proceeding, let us notice that, since words representing the same element of $M$ must have the same length, we have a natural concept of length for the elements of $M$.

Then, given $a \in A$, there exist $x, y \in M$ such that $x a y \in \operatorname{Fix}(\varphi)$. Thus, since $x a y=(x a y) \varphi^{n}$ for all $n \geqslant 1$, the element $a \varphi^{n}$ is a factor of $x a y$, and $\left|a \varphi^{n}\right| \leqslant|x a y|$ for all $n \geqslant 1$. Therefore $\left\{a, a \varphi, a \varphi^{2}, \ldots\right\}$ must be finite.

It follows that, for every $a \in A$, there exist $m_{a} \geqslant 0$ and $p_{a} \geqslant 1$ such that $a \varphi^{m_{a}+p_{a}}=a \varphi^{m_{a}}$. For any integers $m$ and $p$ such that $m \geqslant m_{a}$ and $p$ is positive multiple of $p_{a}$ for all $a \in A$, we have $a \varphi^{m+p}=a \varphi^{m}$ for every $a \in A$. Thus, we may take such an $m$ and such a $p$ satisfying $m=p-1$, yielding $a \varphi^{2 p-1}=a \varphi^{p-1}$ for all $a \in A$. Hence, for every $u \in M$,

$$
u \varphi^{2 p-1}=u \varphi^{p-1}
$$

and, in general, $u \varphi^{j p-1}=u \varphi^{p-1}$ for every $j \geqslant 1$. It follows that

$$
u \varphi^{n}=1 \Rightarrow u \varphi^{p-1}=1
$$

for all $u \in M$ and $n \geqslant 1$. We now prove that

$$
\operatorname{Fix}(\varphi)=\left(\operatorname{Fix}\left(\varphi^{p-1}\right)\right) \varphi^{p}
$$


If $u \in \operatorname{Fix}(\varphi)$, then $u=u \varphi^{p-1}=u \varphi^{p}$ yields $u \in\left(\operatorname{Fix}\left(\varphi^{p-1}\right)\right) \varphi^{p}$. Conversely, let $v \in \operatorname{Fix}\left(\varphi^{p-1}\right)$ and $u=v \varphi^{p}$. Then, in view of (27), $u \varphi=v \varphi^{p+1}=v \varphi^{p-1} \varphi^{p+1}=v \varphi^{2 p}=v \varphi^{p}=u$, and so (29) holds.

Thus $\operatorname{rk}_{\mathcal{M}}(\operatorname{Fix}(\varphi)) \leqslant \operatorname{rk}_{\mathcal{M}}\left(\operatorname{Fix}\left(\varphi^{p-1}\right)\right)$. By replacing $\varphi$ by $\varphi^{p-1}$, in view of $(28)$ this allows us to assume that

$$
a \varphi^{n}=1 \Rightarrow a \varphi=1
$$

for all $a \in A$ and $n \geqslant 1$. Let

$$
A_{0}=A \cap 1 \varphi^{-1} \quad \text { and } \quad A_{1}=A \backslash A_{0} .
$$

Then

$$
A_{1} \varphi^{n} \subseteq M \backslash A_{0}^{*}
$$

for every $n \geqslant 1$.

Now we split our discussion into two cases. We consider first the case $A_{0} \neq \emptyset$.

Consider the homomorphism between free monoids $\pi: A^{*} \rightarrow A_{1}^{*}$ which erases the letters of $A_{0}$. Let $M_{1}$ be the monoid defined by the presentation

$$
\left\langle A_{1} \mid\left(a_{1} a_{2}\right) \pi=\left(a_{3} a_{4}\right) \pi\right\rangle .
$$

We claim that (31) is equivalent to some presentation of the form (26). This clearly holds if $\left|\left(a_{1} a_{2}\right) \pi\right|=\left|\left(a_{3} a_{4}\right) \pi\right|$. On the other hand, the facts that $1 \pi^{-1}=A_{0}^{*},\left(a_{1} a_{2}\right) \varphi=\left(a_{3} a_{4}\right) \varphi$ and in $M$ there is no invertible elements other than 1 imply that $\left(a_{1} a_{2}\right) \pi=1$ if and only if $\left(a_{3} a_{4}\right) \pi=1$. Therefore we are left, in view of left-right symmetry, with the case $a_{1}, a_{3}, a_{4} \in A_{1}$ and $a_{2} \in A_{0}$.

Suppose that $a_{1} \in\left\{a_{3}, a_{4}\right\}$. Then $\left|\left(a_{1} a_{2}\right) \varphi\right|=\left|\left(a_{3} a_{4}\right) \varphi\right|$ implies $a_{i} \varphi=1$ for some $i \in\{3,4\}$, contradicting $a_{i} \in A_{1}$. Hence $a_{1} \notin\left\{a_{3}, a_{4}\right\}$. But then $M_{1}$ is the free monoid on $A_{1} \backslash\left\{a_{1}\right\}$.

Let $\theta: A^{*} \rightarrow M$ and $\theta_{1}: A_{1}^{*} \rightarrow M_{1}$ be the canonical homomorphisms. Since both homomorphisms $\pi \theta_{\left.\right|_{1} ^{*}} \varphi$ and $\theta \varphi$ coincide for letters of $A_{0}$ and $A_{1}$, we have

$$
\pi \theta_{\left.\right|_{1} ^{*}} \varphi=\theta \varphi
$$

As $\operatorname{Ker}(\theta)$ is the congruence generated by the relation $a_{1} a_{2}=a_{3} a_{4}$ and $\left(a_{1} a_{2}\right) \pi \theta_{1}=\left(a_{3} a_{4}\right) \pi \theta_{1}$, there exists a homomorphism $\pi^{\prime}: M \rightarrow M_{1}$ such that

$$
\theta \pi^{\prime}=\pi \theta_{1}
$$

On the other hand, since $\operatorname{Ker}\left(\theta_{1}\right)$ is the congruence generated by the relation $\left(a_{1} a_{2}\right) \pi=\left(a_{3} a_{4}\right) \pi$ and $\left(a_{1} a_{2}\right) \pi \theta_{\left.\right|_{A_{1}^{*}}} \varphi=\left(a_{3} a_{4}\right) \pi \theta_{\left.\right|_{1} ^{*}} \varphi$ in view of $(32)$, there exists a homomorphism $\psi: M_{1} \rightarrow M$ such that

$$
\theta_{1} \psi=\theta_{\left.\right|_{A_{1}^{*}}} \varphi
$$

We show that

$$
\pi^{\prime} \psi=\varphi
$$


Indeed, since $\theta$ is onto, (35) follows from

$$
\theta \pi^{\prime} \psi=\pi \theta_{1} \psi=\pi \theta_{\left.\right|_{1} ^{*}} \varphi=\theta \varphi,
$$

where these equalities come from (33), (34) and (32), respectively.

We show next that

$$
\operatorname{Fix}(\varphi)=\left(\operatorname{Fix}\left(\psi \pi^{\prime}\right)\right) \psi
$$

Let $v \in A^{*}$ be such that $v \theta \in \operatorname{Fix}(\varphi)$. By (32) and (34), we have

$$
v \theta=v \theta \varphi=v \pi \theta \varphi=v \pi \theta_{1} \psi
$$

Now (34), (32) and (33) yield

$$
\left(v \pi \theta_{1}\right) \psi \pi^{\prime}=v \pi \theta \varphi \pi^{\prime}=v \theta \varphi \pi^{\prime}=v \theta \pi^{\prime}=v \pi \theta_{1},
$$

hence $v \pi \theta_{1} \in \operatorname{Fix}\left(\psi \pi^{\prime}\right)$ and $\operatorname{Fix}(\varphi) \subseteq\left(\operatorname{Fix}\left(\psi \pi^{\prime}\right)\right) \psi$.

Conversely, let $v \in A_{1}^{*}$ be such that $v \theta_{1} \in \operatorname{Fix}\left(\psi \pi^{\prime}\right)$. Then (35) yields

$$
v \theta_{1} \psi \varphi=v \theta_{1} \psi \pi^{\prime} \psi=v \theta_{1} \psi
$$

hence $\left(\operatorname{Fix}\left(\psi \pi^{\prime}\right)\right) \psi \subseteq \operatorname{Fix}(\varphi)$ and $(36)$ holds.

Now we may apply the induction hypothesis to the endomorphism $\psi \pi^{\prime}$ of $M_{1}$ to get

$$
\operatorname{rk}_{\mathcal{M}}\left(\operatorname{Fix}\left(\psi \pi^{\prime}\right)\right) \leqslant\left|A_{1}\right|
$$

Therefore (36) yields

$$
\operatorname{rk}_{\mathcal{M}}(\operatorname{Fix}(\varphi)) \leqslant \operatorname{rk}_{\mathcal{M}}\left(\operatorname{Fix}\left(\psi \pi^{\prime}\right)\right) \leqslant\left|A_{1}\right|<|A|
$$

and the case $A_{0} \neq \emptyset$ is settled.

We assume now that $A_{0}=\emptyset$. By $(30)$, we have $|a \varphi| \geqslant 1$ for every $a \in A=A_{1}$. Recall that we are considering the case $A=A_{f}$. Thus, if there exists $a \in A$ such that $|a \varphi|>1$, then $|u \varphi|>|u|$ for every $u \in M$ that has $a$ as a factor, contradicting the fact that $a \in A_{f}$. It follows that

$$
|a \varphi|=1 \text { for every } a \in A \text {. }
$$

Let $B=A \backslash\left\{a_{1}, \ldots, a_{4}\right\}$ and $C=\left\{a_{1}, \ldots, a_{4}\right\}$. Any word $u$ of $A^{*}$ can be factorized in a unique way in the form $u=w_{0} u_{1} w_{1} \cdots u_{n} w_{n}$, where $n \geqslant 0, w_{0}, w_{n} \in B^{*}, w_{1}, \ldots, w_{n-1} \in B^{+}$and $u_{1}, \ldots, u_{n} \in C^{*}$. A word $v$ of $A^{*}$ represents the same element of $M$ as such a word $u$ if and only if $v=w_{0} v_{1} w_{1} \cdots v_{n} w_{n}$, where $v_{1}, \ldots, v_{n} \in C^{*}$ are such that $u_{i}$ and $v_{i}$ represent the same element of $M$ for every $i=1, \ldots, n$. Then, in view of (37) and that every letter of $B$ occurs in a fixed point of $\varphi$, we have

$$
a \varphi=a \text { for every } a \in B,
$$

and $C \varphi \subseteq C$. Let $M_{C \varphi}$ be the submonoid of $M$ generated by $C \varphi$. Then

$$
\operatorname{Fix}(\varphi)=\left(B \cup \operatorname{Fix}\left(\varphi_{\left.\right|_{M_{C}}}\right)\right)^{*} .
$$


If $C \varphi \neq C$, by the induction hypothesis it follows that

$$
\operatorname{rk}_{\mathcal{M}}(\operatorname{Fix}(\varphi))=|B|+\operatorname{rk}_{\mathcal{M}}\left(\operatorname{Fix}\left(\varphi_{\left.\right|_{M_{C \varphi}}}\right)\right) \leqslant|B|+|C \varphi|<|B|+|C|=|A| .
$$

Suppose now that $C \varphi=C$. Then $\varphi$ induces a permutation on $A$. If $\varphi$ is the identity of $M$, then $\operatorname{Fix}(\varphi)=M$, whence $\operatorname{rk}_{\mathcal{M}}(\operatorname{Fix}(\varphi))=|A|$. If $\varphi$ is not the identity of $M$ and the words $a_{1} a_{2}$ and $a_{3} a_{4}$ are equal, then $M$ is the free monoid on $A$, and $\operatorname{Fix}(\varphi)=B^{*}$, whence $\operatorname{rk}_{\mathcal{M}}(\operatorname{Fix}(\varphi))=|B|<|A|$. We proceed under the assumption that $\varphi$ is not the identity of $M$ and that the words $a_{1} a_{2}$ and $a_{3} a_{4}$ are distinct. In view of (38), and using left-right symmetry, we may assume that $a_{1} \varphi \neq a_{1}$. Suppose that $a_{1} \varphi \neq a_{3}$. Then, from $\left(a_{1} a_{2}\right) \varphi=\left(a_{3} a_{4}\right) \varphi$ and the definition of $M$, we have $a_{1} \varphi=a_{3} \varphi$ and $a_{2} \varphi=a_{4} \varphi$, whence $a_{1}=a_{3}$ and $a_{2}=a_{4}$, a contradiction. Hence $a_{1} \varphi=a_{3}$. It follows that $a_{1} \neq a_{3}$. From the fact that $\varphi$ is induces a permutation on $A$ and the definition of $M$, the homomorphism $\varphi$ must permute $a_{1}$ with $a_{3}$ as well as $a_{2}$ with $a_{4}$. We split our discussion into two cases.

In the case that $a_{2}=a_{4}$ it is easy to check that

$$
\operatorname{Fix}(\varphi)=\left(\left(A \backslash\left\{a_{1}, a_{3}\right\}\right) \cup\left\{a_{1} a_{2}\right\}\right)^{*} .
$$

Therefore $\operatorname{rk}_{\mathcal{M}}(\operatorname{Fix}(\varphi))<|A|$ in this case.

Assume now that $a_{2} \neq a_{4}$. Then our defining relation of $M$ must be of the form $a b=c d$ or $a b=b a$ or $a^{2}=b^{2}$, with $a, b, c, d$ distinct. If it is $a b=c d$ or $a b=b a$, it is easy to check that

$$
\operatorname{Fix}(\varphi)=(B \cup\{a b\})^{*},
$$

yielding $\operatorname{rk}_{\mathcal{M}}(\operatorname{Fix}(\varphi))<|A|$. Let us consider the case where the relation is $a^{2}=b^{2}$. The rewriting system (see [5] for details)

$$
\left\{b^{2} \longrightarrow a^{2}, b a^{2} \longrightarrow a^{2} b\right\}
$$

is noetherian (there are no infinite chains of reductions since the lexicographic order is a well-order) and locally confluent (since the unique overlappings between relators are those of the form $b b^{2}=b^{2} b$ and $b\left(b a^{2}\right)=b^{2} a^{2}$, and both lead to commutative diagrams such as
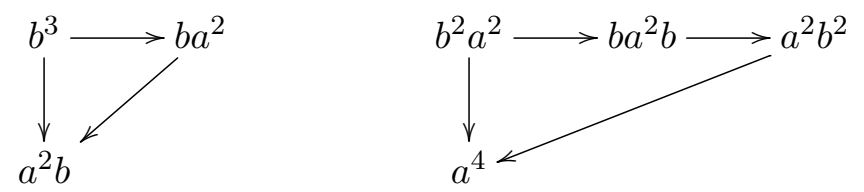

The rewriting system is then confluent and the set of irreducible words is a set of normal forms for $M$. The irreducible words are those of the form $w_{0} v_{1} w_{1} \cdots v_{n} w_{n}$, where $n \geqslant 0, w_{0}, w_{n} \in B^{*}$, $w_{1}, \ldots, w_{n-1} \in B^{+}$and $v_{1}, \ldots, v_{n} \in a^{*}(b a)^{*}\{1, b\}$.

Now, it is easy to check that

$$
\operatorname{Fix}(\varphi)=\left(B \cup\left\{a^{2}\right\}\right)^{*},
$$

and $\operatorname{rk}_{\mathcal{M}}(\operatorname{Fix}(\varphi))=|B|+1<|A|$ as required.

Now, we may conclude the following. 
Corollary 3.5. Any monoid (respectively semigroup) defined by a finite presentation of the form

$$
\left\langle A \mid a_{1} a_{2}=a_{3} a_{4}\right\rangle,
$$

with $a_{1}, \ldots, a_{4} \in A$ not necessarily distinct, is in $\mathbf{U E}(\mathcal{M})$ (respectively $\mathbf{U E}(\mathcal{M})$ ).

Proof. The statement for monoids follows directly from Theorem 3.4 . Suppose that $S$ is a semigroup defined by such a (semigroup) presentation $\left\langle A \mid a_{1} a_{2}=a_{3} a_{4}\right\rangle$. Since $S$ does not have an identity, the monoid $S^{1}$ is also defined by the (monoid) presentation $\left\langle A \mid a_{1} a_{2}=a_{3} a_{4}\right\rangle$. Then any (semigroup) endomorphism $\varphi$ of $S$ can be naturally extended to a (monoid) endomorphism $\varphi_{1}$ of $S^{1}$. For such endomorphisms, we have $\operatorname{Fix}\left(\varphi_{1}\right)=\operatorname{Fix}(\varphi) \cup\{1\}$, whence $\operatorname{rk}(\operatorname{Fix}(\varphi))=\operatorname{rk}_{\mathcal{M}}\left(\operatorname{Fix}\left(\varphi_{1}\right)\right)$. The desired conclusion now follows from Theorem 3.4 .

Contrarily to what happens in free groups, Rodaro and the third author proved [19, Theorem 3.10 and Corollary 3.11] that any nontrivial finitely generated free inverse monoid has automorphisms $\varphi$ such that $\operatorname{Fix}(\varphi)$ is not finitely generated. Hence nontrivial finitely generated free inverse monoids are not in $\mathbf{U A}(\mathcal{M})$, and thus not in $\mathbf{U E}(\mathcal{M})$ either.

Next we will see some relationships between the classes $\operatorname{Tak}(\mathcal{V})$ and the classes UA and UE.

A simple adaptation of an argument known for groups (see e.g. the proof of [1, Theorem 5.1]) allows us to prove the following:

Theorem 3.6. Let $\mathcal{V}$ be one of the varieties of type $(2)$ or $(2,1)$ considered in Section 1 and let $S \in \operatorname{Tak}(\mathcal{V})$.

(i) If $S \in \mathbf{U A}$, then $\operatorname{Per}(\varphi)$ is finitely generated for every $\varphi \in \operatorname{Aut}(S)$.

(ii) If $S \in \mathbf{U E}$, then $\operatorname{Per}(\varphi)$ is finitely generated for every $\varphi \in \operatorname{End}(S)$.

Proof. (i) If $S \in \mathbf{U A}$, then there exists some $N \in \mathbb{N}$ such that

$$
\forall \psi \in \operatorname{Aut}(S), \quad \operatorname{rk}(\operatorname{Fix}(\psi)) \leqslant N .
$$

Let $\varphi \in \operatorname{Aut}(S)$. It is easy to see that

$$
m \mid n \Rightarrow \operatorname{Fix}\left(\varphi^{m}\right) \leqslant \operatorname{Fix}\left(\varphi^{n}\right)
$$

holds for all $m, n \geqslant 1$. Hence we have an ascending chain of subsemigroups of $S$ of the form

$$
\operatorname{Fix}(\varphi) \leqslant \operatorname{Fix}\left(\varphi^{2 !}\right) \leqslant \operatorname{Fix}\left(\varphi^{3 !}\right) \leqslant \cdots
$$

By 39 , we have $\operatorname{rk}\left(\operatorname{Fix}\left(\varphi^{n !}\right)\right) \leqslant N$ for every $n \geqslant 1$. Since $S \in \operatorname{Tak}(\mathcal{V})$, there exists some $k \geqslant 1$ such that $\operatorname{Fix}\left(\varphi^{n !}\right)=\operatorname{Fix}\left(\varphi^{k !}\right)$ for every $n \geqslant k$. In view of 40$)$, we get

$$
\operatorname{Per}(\varphi)=\bigcup_{n \geqslant 1} \operatorname{Fix}\left(\varphi^{n}\right)=\bigcup_{n \geqslant 1} \operatorname{Fix}\left(\varphi^{n !}\right)=\operatorname{Fix}\left(\varphi^{k !}\right) .
$$

Therefore $\operatorname{rk}(\operatorname{Per}(\varphi))=\operatorname{rk}\left(\operatorname{Fix}\left(\varphi^{k !}\right)\right) \leqslant N$ by 39$)$ and so $\operatorname{Per}(\varphi)$ is finitely generated.

(ii) Similar. 
We remark that, even for $S \in \operatorname{Tak}(\mathcal{V})$, the conditions $S \in \mathbf{U A}$ or $S \in \mathbf{U E}$ are far from necessary to get finitely generated periodic subalgebras. For instance, it follows from the results in [19, Section 3] that $\operatorname{Fix}(\psi)$ is not finitely generated when $M$ is the free monogenic inverse monoid and $\psi \in \operatorname{Aut}(M)$ sends the generator $a$ to its inverse $a^{-1}$. However, by [19, Theorem 3.8], Per $(\varphi)$ is finitely generated for every endomorphism $\varphi$ of a free inverse monoid of finite rank.

A straightforward adaptation of the proof of [1, Corollary 5.2] yields the following corollary. We include the (short) proof for completeness.

Corollary 3.7. Let $\mathcal{V}$ be one of the varieties of type $(2)$ or $(2,1)$ considered in Section 1 and let $S \in \operatorname{Tak}(\mathcal{V})$.

(i) If $S \in \mathbf{U A}$ and $\varphi \in \operatorname{Aut}(S)$, then there exists a constant $R_{\varphi}>0$ such that every $a \in \operatorname{Per}(\varphi)$ has period less or equal to $R_{\varphi}$.

(ii) If $S \in \mathbf{U E}$ and $\varphi \in \operatorname{End}(S)$, then there exists a constant $R_{\varphi}>0$ such that every $a \in \operatorname{Per}(\varphi)$ has period less or equal to $R_{\varphi}$.

Proof. (i) By Theorem 3.6, we may write $\operatorname{Per}(\varphi)=\left\{a_{1}, \ldots, a_{r}\right\}^{+}$. Let $R_{\varphi}$ denote the least common multiple of the periods of the elements $a_{1}, \ldots, a_{r}$. Let $a \in \operatorname{Per}(\varphi)$. Then there exist $i_{1}, \ldots, i_{n} \in\{1, \ldots, r\}$ such that $a=a_{i_{1}} \cdots a_{i_{n}}$. It follows that

$$
a \varphi^{R_{\varphi}}=\left(a_{i_{1}} \cdots a_{i_{n}}\right) \varphi^{R_{\varphi}}=\left(a_{i_{1}} \varphi^{R_{\varphi}}\right) \cdots\left(a_{i_{n}} \varphi^{R_{\varphi}}\right)=a_{i_{1}} \cdots a_{i_{n}}=a,
$$

hence $a$ has period less or equal to $R_{\varphi}$.

(ii) Similar.

We note that Theorem 3.6 and Corollary 3.7 also hold for $\mathcal{V}=\mathcal{M}$ by replacing $\mathbf{U A}$ and UE by $\mathbf{U A}(\mathcal{M})$ and $\mathbf{U E}(\mathcal{M})$, respectively.

Now we get the following result:

Theorem 3.8. Let $S \in \mathcal{C S} \cup \mathcal{C}$ be finitely generated with all $\mathcal{H}$-classes in $\operatorname{Tak}(\mathcal{G}) \cap \mathbf{U E}$. Then:

(i) $\operatorname{Per}(\varphi)$ is finitely generated for every $\varphi \in \operatorname{End}(S)$;

(ii) for every $\varphi \in \operatorname{End}(S)$, there exists a constant $R_{\varphi}>0$ such that every a $\in \operatorname{Per}(\varphi)$ has period less or equal to $R_{\varphi}$.

Proof. By Theorems 2.12 or 2.14, we have accordingly $S \in \operatorname{Tak}(\mathcal{C S})$ or $S \in \operatorname{Tak}(\mathcal{C})$. By Proposition 3.2 and Theorem 3.6(ii), $\operatorname{Per}(\varphi)$ is finitely generated for every $\varphi \in \operatorname{End}(S)$. In view of Corollary 3.7(ii), we may now obtain (ii).

Similarly, in view of Corollaries 3.5 and 3.7(ii) and Theorem 3.6(ii), we get also

Theorem 3.9. Let $S$ be the monoid (respectively semigroup) defined by a finite presentation of the form

$$
\left\langle A \mid a_{1} a_{2}=a_{3} a_{4}\right\rangle,
$$

with $a_{1}, \ldots, a_{4} \in A$ not necessarily distinct. Then: 
(i) $\operatorname{Per}(\varphi)$ is finitely generated for every $\varphi \in \operatorname{End}(S)$;

(ii) for every $\varphi \in \operatorname{End}(S)$, there exists a constant $R_{\varphi}>0$ such that every $a \in \operatorname{Per}(\varphi)$ has period less or equal to $R_{\varphi}$.

Note that Example 3.3 shows that Theorem 3.9 cannot be generalized to presentations with a relation $u=v$ such that $|u|=|v|=3$.

\section{Acknowledgements}

This work was mostly developed within the activities of FCT's (Fundação para a Ciência e a Tecnologia's) project PEst-OE/MAT/UI0143/2013-14 of the Centro de Álgebra da Universidade de Lisboa (CAUL), that supported the visit of the first author to CAUL, and it was concluded within the FCT project of CEMAT.

The third author acknowledges support from the European Regional Development Fund through the programme COMPETE and the Portuguese Government through FCT under the project PEstC/MAT/UI0144/2013.

\section{References}

[1] V. Araújo, P. V. Silva and M. Sykiotis, Finiteness results for subgroups of finite extensions, J. Algebra 423 (2015), 592-614.

[2] L. Bartholdi and P. V. Silva, Rational subsets of groups, Chapter 23 of the handbook $A u-$ toMathA (to appear), arXiv:1012.1532, 2010.

[3] M. Bestvina and M. Handel, Train tracks and automorphisms of free groups, Ann. Math. 135 (1992), 1-51.

[4] O. Bogopolski and K.-U. Bux, Subgroup conjugacy separability for surface groups, preprint, 2013.

[5] R. V. Book and F. Otto, String-Rewriting Systems, Springer-Verlag, New York, 1993.

[6] A. H. Clifford, The free completely regular semigroup on a set, J. Algebra 59 (2) (1979), 434-451.

[7] F. Galvin, Embedding countable groups in 2-generator groups, Amer. Math. Monthly 100 (6) (1993), 578-580.

[8] R. Gray and N. Ruškuc, Green index and finiteness conditions for semigroups, J. Algebra 320 (8) (2008), 3145-3164.

[9] P. A. Grillet, Commutative semigroups, Kluwer Academic Publishers, 2001. 
[10] G. Higman, A finitely related group with an isomorphic proper factor group, J. London Math. Soc. 26 (1951), 59-61.

[11] G. Higman, B. H. Neumann and H. Neumann, Embedding theorems for groups, J. London Math. Soc. 24 (1949), 247-254.

[12] W. Imrich and E. C. Turner, Endomorphisms of free groups and their fixed points, Math. Proc. Cambridge Philos. Soc. 105 (3) (1989), 421-422.

[13] B. A. Jensen and D. W. Miller, Commutative semigroups which are almost finite, Pacific J. Math. 27 (3) (1968), 533-538.

[14] M. V. Lawson, Finite Automata, Chapman \& Hall/CRC, 2003.

[15] R. C. Lyndon and P. E. Schupp, Combinatorial Group Theory, Springer-Verlag, 1977.

[16] W. Magnus, A. Karrass and D. Solitar, Combinatorial Group Theory, John Wiley \& Sons Inc., 1966.

[17] B. H. Neumann and H. Neumann, Embedding theorems for groups, em J. London Math. Soc. 34 (1959), 465-479.

[18] E. Rodaro and P. V. Silva, Fixed points of endomorphisms of trace monoids, Semigroup Forum 89(1) (2014), 266-279.

[19] E. Rodaro and P. V. Silva, On periodic points of free inverse monoid endomorphisms, Internat. J. Algebra Comput. 23(8) (2013), 1789-1804.

[20] W. Y. Sit and M-K. Siu, On the subsemigroups of $\mathbb{N}$, Math. Mag. 48 (1975), 225-227.

[21] M. Takahasi, Note on locally free groups, J. Inst. Polytechnics, Osaka City Univ. ser. A 1(2) (1950), 65-70. 